\section{Transforming the Energy Sys- tem: The Role of Institutions, Interests and Ideas}

Bericht von der " $7^{\text {th }}$ International Summer Academy on Technology Studies“

\section{Deutschlandsberg, Österreich, 27. - 31. August 2007}

\section{von Christian Dieckhoff, ITAS}

\section{Hintergrund}

Bereits zum siebten Mal veranstalteten das Interuniversitäre Forschungszentrum für Technik, Arbeit und Kultur (IFZ, Graz) und die Alpen-Adria-Universität Klagenfurt vom 27. bis 31. August 2007 die ,International Summer Academy on Technology Studies“" auf der Burg Deutschlandsberg in der Steiermark, dieses Mal unter dem Titel ,Transforming the Energy System: The Role of Institutions, Interests and Ideas". Die Veranstaltung, die sich mittlerweile als eine der wichtigsten Konferenzen der Science- and Technology Studies (STS) etabliert hat, bot ungefähr 40 Teilnehmern die Gelegenheit, ihre Forschungen zum Wandel des Energiesystems vor dem Hintergrund sozialer und ökologischer Nachhaltigkeitsziele vorzustellen.

Dieses weit gefasste Forschungsfeld wurde von den Veranstaltern im Vorfeld zugespitzt: "Institutions" - the Challenges of Mesoand Macro-Level Transition, "Interests" - the Negotiation of Goals and the Alignment of Actors, "Ideas" - the Social Dynamics of Visions and Expectations. Im Zentrum der Konferenz standen weniger das ökonomischtechnische Energiesystem und die sich aus dieser Perspektive ergebenden Möglichkeiten seiner Wandlung, sondern vielmehr die gesellschaftlichen und politischen Bedingungen, unter denen diese Transformation geschehen kann. Die Identifikation von relevanten Institutionen und gesellschaftlichen Gruppen, ihre spezifischen Gestaltungsmöglichkeiten und Interessen waren deshalb Kernthemen der Konferenz. Den theoretischen Hintergrund bildeten Ansätze des Transition-Managements (TM), wobei unter ,Transitions“ (auch Systeminnova- tionen) Transformationsprozesse verstanden werden, in denen sich die Gesellschaft oder ein komplexes gesellschaftliches Subsystem in fundamentaler Weise über einen längeren Zeitraum hinweg wandelt. TM zielt dann darauf $a b$, diese Transformationen ,zu erforschen, zu leiten und $\mathrm{zu}$ unterstützen" (Rotmans et al. 2000, S. 3, Übersetzung des Autors).

\section{Ausgewählte Vorträge}

Boelie Elzen (University of Twente) stellte mit seinem Vortrag ,Exploring future transition pathways - The Socio-Technical Scenario approach" eine Methode vor, die neben den technischen vor allem auch die gesellschaftlichen Dimensionen von Transitions berücksichtigt. Diese Perspektive würde in anderen SzenarioMethoden - laut Elzen - häufig fehlen. Aufbauend auf der Mehr-Ebenen-Perspektive und hieraus abgeleiteter charakteristischer Transitionspfade werden hierbei zunächst ,SzenarioSkelette" erstellt, die anschließend gemäß einer spezifischen „Logik“ in unterschiedliche Geschichten umgeschrieben werden. Laut Elzen sind bei diesem Vorgehen zwei Aspekte zentral: der narrative Ansatz sowie eine enge Abstimmung mit dem Auftraggeber der Studie.

Unter dem Titel „How do innovation system dynamics explain successes and failures of emergent technological trajectories in the Dutch transportation fuels sector?" stellte Roald Suurs (University Utrecht) sein Promotionsprojekt vor. Gegenstand seiner Untersuchung ist die Entwicklung von Biokraftstoffen in den Niederlanden, wobei die simultane Entwicklung von Kraftstoffen erster und zweiter Generation von besonderem Interesse ist. Den theoretischen Hintergrund seiner Arbeit bildet der Technological Innovation Systems Approach (Carlsson, Stankiewicz 1991) und die darauf aufbauenden Systemfunktionen, die Schlüsselprozesse des technologischen Wandels (z. B. Wissensbildung oder Ressourcenmobilisierung) repräsentieren. Suurs weist diesen Funktionen charakteristische Ereignisse $\mathrm{zu}$, die anhand historischer Daten empirisch nachgewiesen werden. Ziel ist es, daraus Einsichten in typische Muster technologischen Wandels zu gewinnen.

In seinem Vortrag ,Sustainability, foresight and contested futures: exploring visions 
and pathways in the transition to a hydrogen economy" berichtete Malcom Eames (Brunel University) über eine weitere SzenarioMethode, die im Rahmen eines Projektes des „UK Sustainable Hydrogen Energy Consortium" (UKSHEC) entwickelt und angewendet wurde. Ziel dieser Methode war es, eine kleine Anzahl „glaubwürdiger“ Wasserstoff-Zukünfte zu entwickeln. Eine zentrale Rolle spielte dabei die Einbindung von Experten, die in der ersten Phase des Projektes anhand eines StakeholderMapping ausgewählt wurden. In der zweiten Phase wurden zunächst im Rahmen eines Workshops von rund 40 teilnehmenden Experten eigene Visionen einer Wasserstoffwirtschaft entwickelt. Das so erfasste breite Spektrum an Erwartungen wurde anschließend von der Forschungsgruppe zu sechs Visionen verdichtet. Diese „Kernvisionen“ wurden wiederum in der dritten Phase mit Hilfe eines MultiCriteria-Mapping hinsichtlich ihrer jeweiligen Nachhaltigkeit von einem Experten-Panel bewertet, um schließlich in der letzten Phase mögliche Pfade zu formulieren, mit denen die Visionen erreicht werden können. Wichtige Ergebnisse des Projektes sind für Eames unter anderem, dass die Nachhaltigkeit der alternativen Wasserstoff-Zukünfte stark umstritten ist und dabei die Frage der nuklearen Energiegewinnung sowie der Grad der Dezentralisierung Schlüsselkonflikte darstellen.

Audley Genus (University Newcastle) analysierte in seinem Vortrag ,A Critique of Geels' Multi-level Perspective of Technological Transition" die Leistungen und Grenzen der Geels'schen Mehr-Ebenen-Perspektive. Hierbei handelt es sich um eine Erweiterung des MehrEbenen-Konzeptes von Rip und Kemp (1998), das insbesondere in der Tradition von Nelson und Winter (1982) und dem von ihnen eingeführten Begriff der Technological Regimes steht. Geels stellt den langfristigen Wandel großtechnischer Systeme und deren gesellschaftliche Einbettung ins Zentrum seiner Betrachtung und unterscheidet drei, ineinander verschachtelte Level, auf denen sich ein solcher soziotechnologischer Wandel vollzieht: Auf dem Mikrolevel werden technologische Nischen beschrieben, die - vom übrigen Markt geschützt - Raum für radikale Innovationen bieten. Erfolgreiche Innovationen können in das Mesolevel der soziotechnologischen Regime eindringen, diese ver- ändern und von dort aus das Makrolevel der soziotechnologischen Landschaft erreichen und damit gestalten. Als einen wichtigen Beitrag benannte Genus die koevolutionäre Orientierung des Ansatzes, in dem neben technischen auch gesellschaftliche Aspekte von Innovation sowie deren Wechselwirkungen berücksichtigt würden. Ein Manko sei dagegen für ihn, dass der Ansatz nicht falsifizierbar sei; es sei unmöglich, Transitions beliebig $\mathrm{zu}$ definieren, ohne die Theorie selbst dabei zu berühren. Dies zeige sich in bisherigen Fallstudien insbesondere bei der Identifikation von Start- und Endpunkten der beobachteten Transitions, die an Ereignissen unterschiedlichen Typs festgemacht seien und damit insbesondere von der Interpretation des Forschers abhingen. Als eine Voraussetzung für die Weiterentwicklung der Mehr-Ebenen-Perspektive betonte Genus vor allem die Notwendigkeit eines sorgfältigeren und transparenteren Umgangs mit dem Ansatz und sah Anlass, gerade die Schwierigkeiten bei seiner Anwendung zum Gegenstand der Diskussion zu machen.

Harald Rohracher (IFZ, Graz) schloss die Sommerakademie mit einer kurzen Zusammenfassung und verbuchte als einen wichtigen Gewinn der Veranstaltung, dass die Vorträge und Diskussionen eine Sensibilisierung für die Möglichkeiten und Grenzen der eigenen Methoden bewirkt hätten. Als eine Hauptaufgabe für die STS-Community betonte er die Notwendigkeit, sich Klarheit über das Konzept der Nachhaltigkeit zu verschaffen.

Dieser Vorschlag kann hier nur unterstützt werden, denn tatsächlich fiel auf, dass das Wort Nachhaltigkeit in vielen Präsentationen zwar verwendet, aber in nur wenigen Fällen erklärt wurde, was darunter verstanden wird oder an wen der jeweilige Ansatz Ziele der Nachhaltigkeit adressiert. Dass dies gleichfalls in der Mehrzahl der Diskussionen unbeachtet blieb, verwundert insbesondere deshalb, weil in einem der ersten Vorträge Armin Grunwald (ITAS, Forschungszentrum Karlsruhe) unter dem Titel ,Sustainable Development as a challenge for transforming the energy system" umfassende Anregungen hierzu gab. 


\section{Abschließende Bemerkungen}

Wie zahlreiche Vorträge zeigten, hatte als jüngere theoretische Entwicklung im Feld der TM für die diesjährige Sommerakademie Geels MehrEbenen-Perspektive (Geels 2002) eine herausragende Bedeutung. Die damit verbundene Vorstellung von Transitions als Innovationspfade und deren Durchlaufen der drei zuvor beschriebenen Ebenen war für viele Diskussionen leitend, die Nützlichkeit des Ansatzes im Sinne einer gemeinsamen Heuristik sei hier deshalb betont. Um jedoch als Theorie zur Erklärung von Innovationen beitragen zu können, ist - im Sinne der Kritik von Genus - eine grundlegende Ausarbeitung des Ansatzes notwendig.

Mit den hier vorgestellten Vorträgen wurde sicherlich nur eine von vielen Diskussionslinien der Sommerakademie nachgezeichnet, unerwähnt blieb beispielsweise die Präsentation einer Vielzahl von Fallstudien, in denen neben nationalen oder regionalen institutionellen Strukturen auch konkrete Technologien thematisiert wurden. Vor allem die vielen Doktorandenvorträge, die rund die Hälfte aller Präsentationen ausmachten, gaben der Sommerakademie einen sehr offenen, lebhaften und produktiven Charakter.

\section{Literatur}

Carlsson, B.; Stankiewicz, R., 1991: On the Nature, Function and Composition of Technological Systems. Journal of Evolutionary Economics 1/2 (1991), pp. 93-118

Geels, F.W., 2002: Technological Transitions as Evolutionary Reconfiguration Processes: A MultiLevel Perspective and a Case-Study. In: Research Policy 31/8-9 (2002), pp. 1257-1274

Nelson, R.R.; Winter, S.G., 1982: An Evolutionary Theory of Economic Change. Cambridge, MA

Rip, A.; Kemp, R., 1998: Technological Change. In: Rayner, S.; Malone, E.L. (eds.): Human Choice and Climate Change. Columbus, Ohio, pp. 327-399

Rotmans, J.; Kemp, R.; van Asselt, M. et al., 2000: Transitions \& Transition Management. For a Low Emission Energy Supply. A Study for the $4^{\text {th }} \mathrm{Na}$ tional Environmental Policy Plan (NMP4). Maastricht; http://www.icis.unimaas.nl/projects/nmp4/ downs/00_35_ab.pdf

)

\section{Integrating Natural and Social Sciences for Sustainability} Tagungsbericht von der ", $7^{\text {th }}$ International Conference of the European Society for Ecological Economics"

\section{Leipzig, 5. - 8. Juni 2007}

\section{von Jürgen Kopfmüller, ITAS, und Fred Luks, Österreichisches Institut für Nachhaltige Entwicklung, Wien}

Unter dem Titel „Integrating Natural and Social Sciences for Sustainability" lud die Europäische Gesellschaft für Ökologische Ökonomie (European Society for Ecological Economics - ESEE) vom 5. bis 8. Juni $2007 \mathrm{zu}$ ihrer siebten internationalen Konferenz ins Helmholtz-Zentrum für Umweltforschung (UFZ) nach Leipzig ein. Die Veranstaltung, an der rund 500 Personen vorwiegend WissenschaftlerInnen - aus der ganzen Welt teilnahmen, wurde gemeinsam von der ESEE, dem UFZ, der Vereinigung für Ökologische Ökonomie (VÖÖ) und der Vereinigung für Ökologische Wirtschaftsforschung (VÖW) organisiert. ${ }^{1}$

Der Tagungstitel „Integrating Natural and Social Sciences for Sustainability" gibt einerseits das Selbstverständnis der Ökologischen Ökonomie als einer inter- und transdisziplinär wirkenden Nachhaltigkeitswissenschaft wieder. So versteht sich die ESEE nicht nur als Vereinigung von ÖkonomInnen, sondern als eine wissenschaftliche Organisation, die disziplinenübergreifend das Nachhaltigkeitsleitbild erforschen und seine Umsetzung voranbringen will. Andererseits bringt der Tagungstitel den Integrationsanspruch einiger WissenschaftlerInnen bzw. die Integrationsforderung an die Wissenschaft selbst zum Ausdruck, die der Beobachtung geschuldet ist, dass Natur- und Sozialwissenschaften nach wie vor kaum integrativ zusammenwirken, sondern im Gegenteil oft unvermittelt nebeneinander stehen.

$\mathrm{Ob}$ die Veranstaltung und die dort präsentierten Beiträge diesen sehr hohen Anspruch der Integration wirklich erfüllen konnten, ist nicht zuletzt eine Frage der Perspektive. Zu beobachten war jedenfalls auch hier, dass BiologInnen eben hauptsächlich biologisch argumentieren, ÖkonomInnen im Wesentlichen wirtschaftlich 